\title{
Engineering the light coupling between metalens and photonic crystal resonators for robust on-chip microsystems
}

\author{
Yahui Xiao $\odot$, Zi Wang, Feifan Wang, Hwaseob Lee $\odot$, Thomas Kananen, \\ and Tingyi Gu* \\ University of Delaware, Department of Electrical and Computer Engineering, Newark, \\ Delaware, United States
}

\begin{abstract}
We designed an on-chip transformative optic system with a broadband metalens coupler on a foundry compatible silicon photonic platform. By adjusting the on-chip metalens' focusing length and mode dimension, the insertion loss between the metalens and the photonic crystal waveguide ( $\mathrm{PhC} \mathrm{WG}$ ) structures is reduced to $2 \mathrm{~dB}$ by matching the mode on the metalens focal plane to the PhC WG mode. Alternatively, the integrated metalens allow for direct coupling from a multi-mode $\mathrm{WG}$ to the $\mathrm{PhC}$ cavity. The on-resonance transmission in a lenscavity-lens microsystem achieves $60 \%$. These micro-systems do not involve any single-mode silicon nanowire WG, and even a suspended $\mathrm{PhC}$ structure can be mechanically robust against vibrations. The proposed microsystem can be a new platform for miniaturized chemical and biosensor applications operating in air or solution environments. (C) The Authors. Published by SPIE under a Creative Commons Attribution 4.0 Unported License. Distribution or reproduction of this work in whole or in part requires full attribution of the original publication, including its DOI. [DOI: 10 .1117/1.JOM.1.2.024001]
\end{abstract}

Keywords: photonic crystals; metalens; mode matching; integrated optics.

Paper 20014 received Sep. 28, 2020; accepted for publication Jan. 13, 2021; published online Mar. 9, 2021.

\section{Introduction}

The highest quality factor $(Q)$ versus modal volume $(V)$ ratio of the photonic crystal $(\mathrm{PhC})$ cavity maximizes the light-matter interactions toward nonlinear optics, quantum optics, and sensing applications. ${ }^{1-8}$ In conventional $\mathrm{PhC}$ circuits, the light is fed through a low-loss channel waveguide (WG) and a PhC WG for coupling into a PhC cavity. ${ }^{9,10}$ The implementation of such a system has a few practical challenges: (1) channel WG is usually fragile and easy to break during the undercut process, which significantly reduces yield during the postprocessing procedures ${ }^{11}$ and makes such a system less practical for any sensing applications; (2) the interface geometry between the channel WG and $\mathrm{PhC}$ WG needs to be carefully engineered to minimize the insertion loss; ${ }^{12-16}$ (3) as a line defect in PhC triangular lattice, the WG geometry needs to be engineered to ensure the overlap between the high-transmission band and $\mathrm{PhC}$ resonance wavelength. Here, we propose a channel WG-free on-chip micro-system composed of a broadband dielectric metalens and a $\mathrm{PhC}$ resonator structure. The critical dimension of such a geometry is more than $100 \mathrm{~nm}$ and is compatible with foundry processing. ${ }^{17,18}$ The microsystem's mechanical robustness and foundry compatibility promise its applications in ultrafast low-power modulators, hybrid lasers, and miniaturized biosensors.

In this work, we compare two channel WG-free designs with and without a PhC WG. The direct coupling between a broadband metalens and PhC L3 cavity can lead to a 0.68 on-resonance transmission and a quality factor of 6100 , compared with the metalens-PhC WG side-coupled L3 cavity structure with the transmission of 0.66 and quality factor of 6600 . The corresponding extinction ratios are 84 and $39 \mathrm{~dB}$, respectively.

*Address all correspondence to Tingyi Gu, tingyigu@udel.edu 


\section{Metalens-PhC WG with Side-Coupled L3 Cavity System}

This section focuses on optimizing the design of the metalens (e.g., focal length and spot size $^{19,20}$ ) and the PhC WG interface geometry to maximize the coupling efficiency. The interface of the $\mathrm{PhC}$ structure is designed by removing etched air holes to generate a $\mathrm{PhC}$ coupler with $38 \mathrm{deg}, 60 \mathrm{deg}, 120 \mathrm{deg}$, and $180 \mathrm{deg}$ (without removing air holes), respectively. Via sweeping the distance between the metalens and the $\mathrm{PhC} \mathrm{WG}$, the optimized position of the PhC structure can be computed from the $2.5 \mathrm{D}$ variational finite difference in time domain simulations.

\subsection{Design Principle of Low-Loss On-Chip Metalens}

Both the metalens and the $\mathrm{PhC}$ are defined on the same device layer on the silicon-on-insulator substrate. The metalens is used as a compact low-loss mode converter. ${ }^{21}$ Here, we use a gradientvarying high-contrast transmit array metalens for wavefront control. The $1 \mathrm{D}$ metalens along the $y$-direction imposes a space-dependent phase shift on the TE polarized impinging light along the $x$-direction. Here, TE polarization is defined as the main $E$ filed component in the $y$-direction, as shown in Fig. 1(b). The input light centered at the wavelength of $1.55 \mu \mathrm{m}$ propagates along the $+x$ direction.

The designed metalens is $10 \mu \mathrm{m}$ wide in the $y$-direction, with a focusing length of $13 \mu \mathrm{m}$ and a spot size of $0.65 \mu \mathrm{m}$, as shown in Fig. 1(a), and the optical intensity distribution of focusing points at the $x-y$ plane in the 250 -nm-thick silicon slab center. The spot size is marked as the fullwidth half-maximum (FWHM) in the cross-section of the mode profile in Fig. 1(c). As the focusing length increases from 4.6 to $25 \mu \mathrm{m}$, the focusing efficiency increases from $29 \%$ to $78 \%$, as shown in Fig. 1(d). The focusing efficiency is defined as the fraction of the input light that passes through a rectangular aperture at the focal plane, with its width equaling three times the spot size and its height being $0.5 \mu \mathrm{m}$. The dashed line in Fig. 1(d) is the FWHM of the electric field intensity distribution in the $\mathrm{PhC} \mathrm{WG}$ as a standard line for mode matching between the metalens
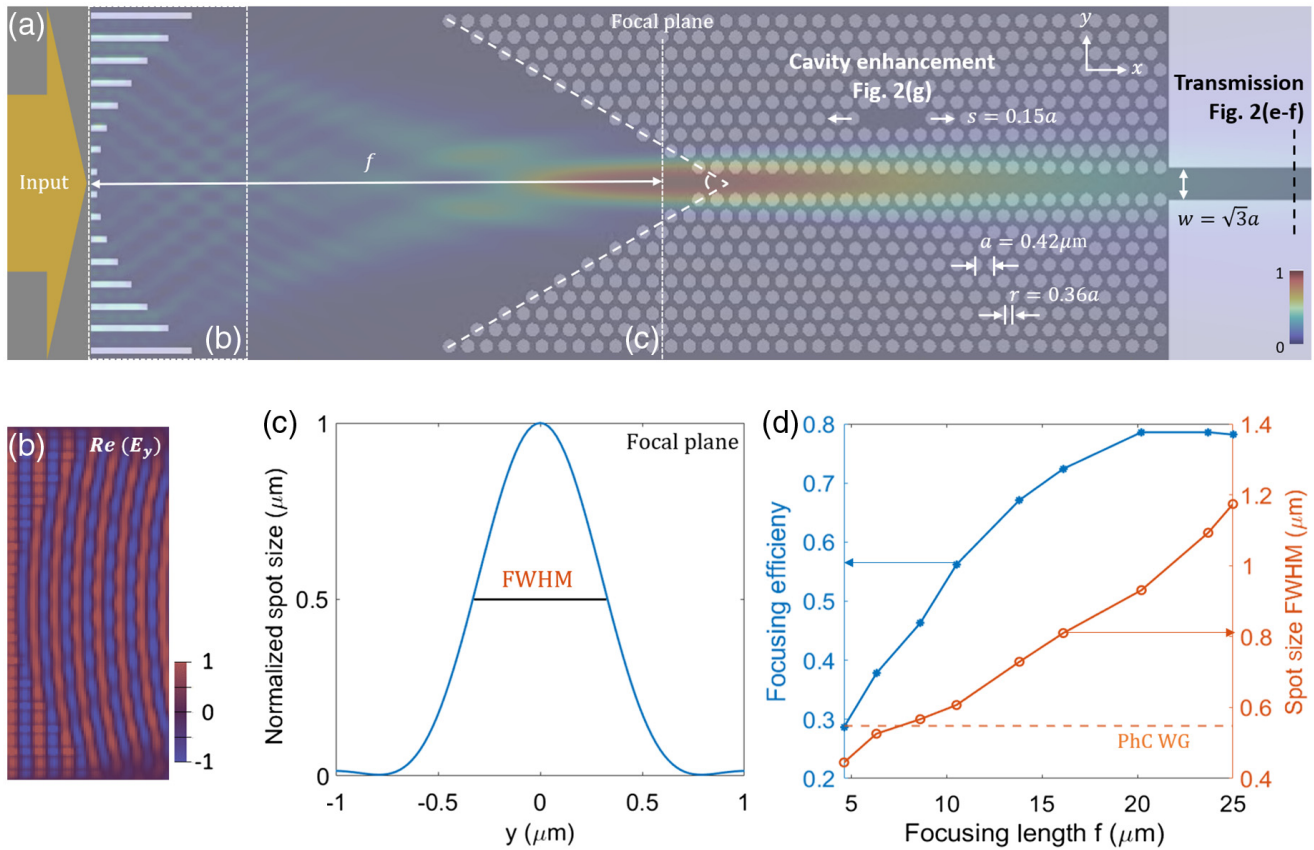

Fig. 1 Mode matching between the integrated metalens and PhC WG. (a) In-plane $(x-y)$ photon intensity of an integrated metalens. The distance between the metalens focal plane and a PhC WG coupler is $L$. (b) Detailed electric field $E_{y}$ as the light passing through the metalens [the dashed box in (a)]. (c) The cross-sectional model profile on the focal plane, with the spot size marked as FWHM. (d) Focusing efficiency (blue line) and FWHM (orange line) of the metalens on the focal plane versus focusing length, compared with the mode width of the W1 PhC WG (orange dash line). 
and $\mathrm{PhC}$ structure. The light with mode profile matching propagates smoothly from the focused Gaussian beam into the periodic $\mathrm{PhC}$ structure. ${ }^{22-24}$

\subsection{PhC WG Coupler}

The structure of W1 PhC WG (relying on a single line-defect in a hexagonal lattice) consists of air holes with a lattice constant $a=0.42 \mu \mathrm{m}$ etched into a silicon slab. The slab thickness is $d=0.6 a$ and the air hole radius is $r=0.36 a$, with the end holes shifted by $s=0.15 a$ in a sidecoupled L3 cavity, as shown in Fig. 1(a). The displaced end holes are primarily to increase the cavity volume for high-index-material $(\mathrm{Si})$ to accumulate more photons, wherein the Bragg reflection and out-of-plane provide the confinement in-plane by total internal reflection. The width of the output channel WG is designed as $w=\sqrt{3} a$ to ensure a high and stable transmission through the PhC WG. The channel WG position is swept to optimize the coupling efficiency between the PhC WG and channel WG. For a single-mode channel WG, the spatial mode profile is independent of the position along the WG direction. However, the spatial mode profile in the PhC WG is changed by the periodic geometry along with the WG. In such a case, the coupling efficiency may change when the PhC WG ends with a different position within its period. $^{25}$

Via integrating with metalens $(f=13 \mu \mathrm{m})$, the input light centered at the wavelength of $1.55 \mu \mathrm{m}$ is focused on a spot size similar to the PhC WG mode, which allows for low insertion loss at the interface. Here, we designed four different $\mathrm{PhC}$ couplers with angles of $38 \mathrm{deg}, 60 \mathrm{deg}$, $120 \mathrm{deg}$, and $180 \mathrm{deg}$ by removing individual air holes at the front edge of the PhC WG to generate an input $\mathrm{PhC}$ taper. ${ }^{26,27}$ Figure 2(a) shows the optimized geometric structure and mode profile at the highest transmission region of PhC WG. The in-plane spot size of the given metalens is $0.65 \mu \mathrm{m}$, which is smaller than the $\mathrm{PhC}$ coupler size $(1.88 \mu \mathrm{m})$ at the coupling plane.

Figures 2(c) and 2(d) show the comparison of three different PhC coupler angles for the total $Q$-factor and enhancement factor of the side coupled L3 cavity. The total $Q$-factor of a resonator is solely limited by out-of-plane radiation that gives a direct measure of the cavity resonance lifetime, which can be re-expressed as $Q_{\text {tot }}=\frac{\lambda_{0}}{\Delta \lambda}$, where $\lambda_{0}$ is the resonant wavelength and $\Delta \lambda$ is the FWHM of the resonance. The enhancement factor implies the electric field intensity in the cavity at resonance.

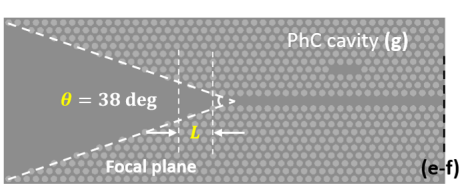

(a)

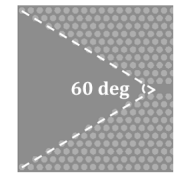

(b)

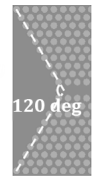

(c)

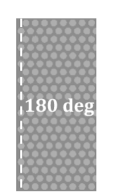

(d)

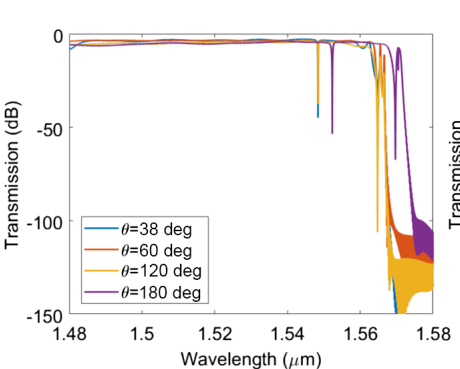

(e)

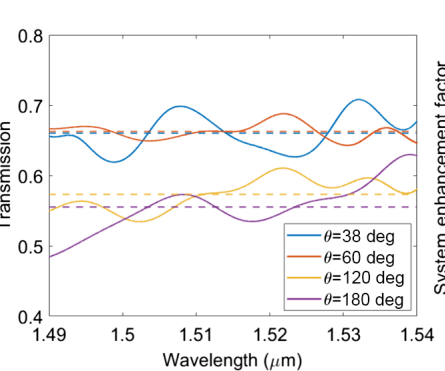

(f)

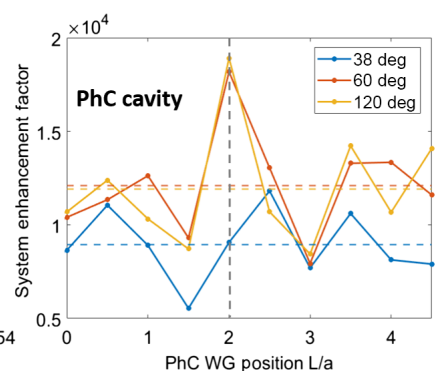

(g)

Fig. 2 Metalens-PhC WG coupler. (a) The geometry of PhC WG coupler with taper angles of $38 \mathrm{deg}$, (b) $60 \mathrm{deg}$, (c) $120 \mathrm{deg}$, and (d) $180 \mathrm{deg}$. (e) Broadband transmission spectra of a metalens-PhC structure in Fig. 1(a), with taper angles in (a)-(d). (f) Detailed comparison of high transmission region. The solid curves are simulation data, and dashed lines are an average value. The ripples are from the FP resonance between the metalens and PhC WG interface. (g) Optical intensity enhancement factor in the L3 PhC cavity side-coupled to the WG compared with the one input to the metalens at different $L$. 
By sweeping the distance between the metalens and PhC WG (coupler angle of $60 \mathrm{deg}$ ) with a step size of $0.5 a$, the system enhancement factor shows an optimized distance between the metalens and PhC WG of $f+L$, where $L=2 a$. However, the PhC WG transmission is not quite sensitive to the changing distance based on sweeping results.

Figure 2(e) shows the transmission of four different PhC WG coupler angles in log scale with resonance dips, where the side-coupled L3 cavities are excited by evanescent coupling from a nearby $\mathrm{PhC} \mathrm{WG} .^{28}$ The band edge of the PhC WGs with the angles of $38 \mathrm{deg}, 60 \mathrm{deg}$, and $120 \mathrm{deg}$ is around $1.57 \mu \mathrm{m}$. However, both the resonance dip and the band edge of these three coupler angles shift to a slightly shorter wavelength, compared with the $180 \mathrm{deg}$ coupler angle (without removing air holes). This phenomenon indicates that changing the PhC WG structure by removing air holes will shift the band edge to a shorter wavelength. Figure 2(f) shows a zoomed transmission part from Fig. 2(e) in linear scale, where the coupler angles of $38 \mathrm{deg}$ and 60 deg have the relatively highest transmission, whereas the one of 60 deg is slightly higher and more stable. Dashed lines are the average transmission value in the same color as the corresponding solid lines. The high transmission comparison shows that the coupler with smaller angles can collect more light and decrease the back reflection by mode matching. Figure $2(\mathrm{~g})$ represents the PhC WG position sweeping results in terms of the system enhancement factor $\left(=\frac{I_{\text {cavity }}}{I_{\text {input }}}\right)$ with the coupler angles of $38 \mathrm{deg}, 60 \mathrm{deg}$, and $120 \mathrm{deg}$, respectively. Coupler angles of $60 \mathrm{deg}$ and $120 \mathrm{deg}$ show a similar trend and reach the optimized PhC WG position with $L=2 a$, marked by a vertical grey dashed line in Fig. $2(\mathrm{~g})$.

\section{Metalens-PhC L3 Cavity System}

Ln cavities represent line defects along the $\Gamma-K$ direction, whereby $n$ adjacent holes are removed from the periodic lattice to localize light along a line. The PhC L3 cavity is the most common configuration line-defect cavity (with three missing holes), with the end holes shifted by $0.15 a$ to obtain a high- $Q$ factor, ${ }^{29}$ as shown in Figs. 3(a) and 3(f). Thus, light is considered to penetrate more inside the mirror and to be reflected perfectly, which means that the cavity edge's electric field profile becomes gentler. Here, we designed a PhC L3 cavity with the same parameters ( $a=0.42 \mu \mathrm{m}, r=0.36 a$, and $s=0.15 a$ ) as the ones of the PhC WG in Sec. 2. Similar to the metalens-PhC WG system, we also compared the PhC L3 cavity with the coupler angles of $38 \mathrm{deg}, 60 \mathrm{deg}$, and $120 \mathrm{deg}$ by integrating with the same metalens $(f=13 \mu \mathrm{m})$. The $y$-direction width of the PhC L3 cavity is designed as the same size of the metalens to increase the coupling region and extend the Bragg reflection in $x-y$ direction. ${ }^{30}$
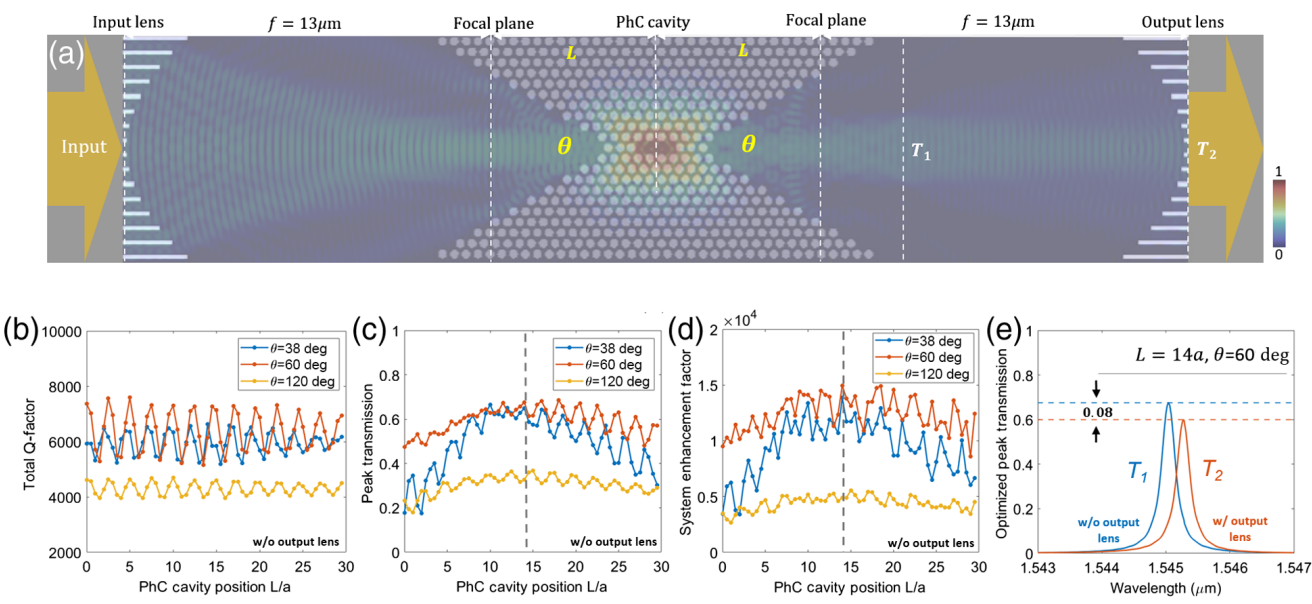

Fig. 3 Direct metalens-PhC L3 cavity coupler. (a) Device geometry of the metalens and PhC L3 cavity. The distance between the metalens and the center of the PhC $L 3$ cavity is $f+L$. (b), (c), and (d) are the sweeping total $Q$ factor, sweeping peak transmission, and sweeping system enhancement factor of the device in (a). (e) Peak transmission comparison spectra of the device in (a) and (f) at an optimized distance, with the PhC L3 cavity coupler angle of 60 deg. 


\subsection{Optimize the Metalens-PhC Cavity Coupler}

Direct coupling between the metalens and $\mathrm{PhC}$ cavity results in a more compact and efficient microsystem [Fig. 3(a)]. The TE polarized light centered at $1.55 \mu \mathrm{m}$ converges through the metalens and impinges on the PhC L3 cavity to excite a fundamental resonant mode ${ }^{31,32}$ at a specific wavelength by the tunneling wave. The simulated result for the total $Q$-factor in Fig. 3(b) shows stable but periodic oscillations, which confirms that, during scanning Fabry-Pérot (FP) resonator length, the Airy distribution originates in the sum of mode profiles of the longitudinal resonator modes. ${ }^{33}$ Based on the sweeping results in terms of the total $Q$-factor, the coupler angle of $60 \mathrm{deg}$ shows the highest average value, slightly higher than the coupler angle of $38 \mathrm{deg}$. Fringes in Fig. 3(a) also imply the standing waves from interference in the FP resonator composed of the metalens and the $\mathrm{PhC}$ structure.

Figure 3(c) plots the transmission of input light at the resonance wavelength of the cavity, with correspondent cavity enhancement factor plotted in Fig. 3(d). As shown in those figures, the optimized $\mathrm{PhC}$ cavity position is placed around $L=14 a$, as marked by the gray dashed lines in Fig. 3(a). The coupler angle of $60 \mathrm{deg}$ (red data curves) shows the highest average data value in peak transmission and system enhancement comparison. The cavity resonance mode profile in log scale shown in Fig. 3(a) may indicate that the mode direction of both the left and right sides of the resonance mode contour appears at $60 \mathrm{deg}$, which matches the PhC L3 cavity coupler angle of $60 \mathrm{deg}$.

\subsection{Metalens-PhC L3 Cavity-Metalens System}

Based on the sweeping results from Fig. 3(a), we selected the PhC cavity coupler angle of $60 \mathrm{deg}$ at the optimized $\mathrm{PhC}$ cavity position $(L=14 a)$ and added a second metalens $(f=13 \mu \mathrm{m})$ symmetric about the center of the PhC L3 cavity based on the ray diagram of double lenses, as shown in Fig. 3(f). We also simulated the bilateral metalens system for the same PhC L3 cavity with the coupler angles of $38 \mathrm{deg}, 60 \mathrm{deg}$, and $120 \mathrm{deg}$. As shown in Fig. 3(e), the highest peak transmission of the system is around 0.6 by the coupler angle of $60 \mathrm{deg}$, which is slightly lower than the one of the metalens-PhC L3 cavity system around 0.68 in Fig. 3(a), mainly because of the transmission loss through the second metalens.

\section{Systems Transmission Comparison}

In this section, we compare the two complete integrating metalens systems. Figure 4 shows the transmissions of the metalens-PhC WG system and the peak transmissions metalensPhC L3 cavity-metalens system, with three coupler angles of $38 \mathrm{deg}, 60 \mathrm{deg}$, and $120 \mathrm{deg}$.

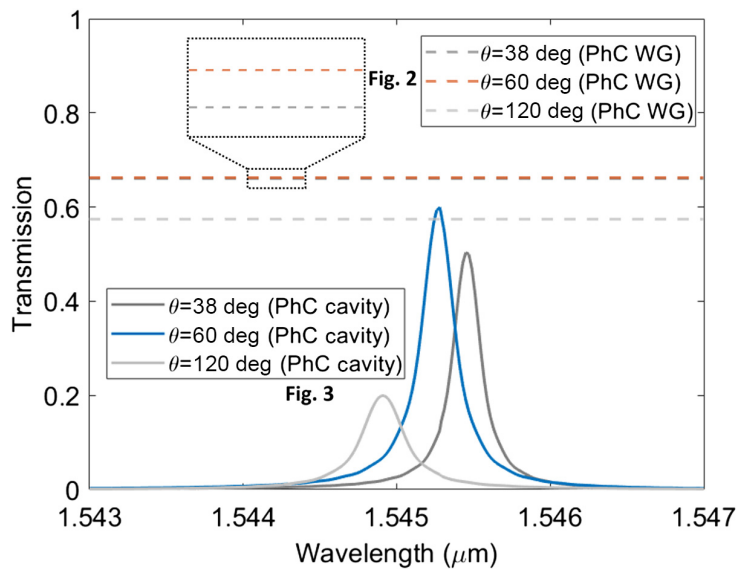

Fig. 4 Transmission spectra of the metalens-PhC microsystems. Dashed lines: metalens-PhC WG system in Fig. 1(a). Solid curves: metalens-PhC L3 cavity-metalens system in Fig. 3(f), with the PhC coupler angles of $38 \mathrm{deg}, 60 \mathrm{deg}$, and $120 \mathrm{deg}$. 
Xiao et al.: Engineering the light coupling between metalens and photonic crystal resonators...

For the transmission of the metalens-PhC WG system, the coupler angle of 38 deg obtains the highest transmission $\sim 0.66$ (marked in the blue curve), whereas for the bilateral metalens- $\mathrm{PhC}$ L3 cavity system, the highest peak transmission $\sim 0.6$ achieved by the $60 \mathrm{deg}$ coupler angle (marked in the red dashed line). For the PhC structure with a coupler angle of $60 \mathrm{deg}$, the total $Q$-factor of the L3 cavity in the bilateral metalens system is around 6000, whereas the total $Q$-factor in the PhC WG side-coupled L3 cavity is about 9500 . For the system enhancement factor, the metalens-PhC WG obtains approximately 18,000, which is higher than the bilateral metalens-PhC L3 cavity with 13,000.

\section{Conclusion}

We demonstrate a low-loss on-chip microsystem based on a broadband metalens and a $\mathrm{PhC}$ resonator through engineering the relative position and geometry of the $\mathrm{PhC}$ and the metalens. Numerical examinations show that the coupler angle of 60 deg defined in the $\mathrm{PhC}$ WG interface leads to the lowest insertion loss $(-4.2 \mathrm{~dB})$. However, in the scheme of the metalens-PhC L3 cavity, the coupler angle of $60 \mathrm{deg}$ obtains the highest total $Q$-factor $(\sim 6100)$ and the system enhancement factor $(\sim 15,000)$. The bilateral metalens system appears to be 0.08 lower than the metalens-PhC system concerning the peak transmission because of the loss from the second metalens.

The designed nanophotonic structures significantly reduce the footprint, reduce the insertion loss, and improve the mechanical robustness of on-chip light coupling into the $\mathrm{PhC}$ structure, which are desired for improving sensitivity in nanophotonic sensors ${ }^{34-36}$ and reducing operation power for PhC-based active silicon photonic components.

\section{Acknowledgments}

This work was supported by the AFOSR Young Investigator Program (FA9550-18-1-0300). $\mathrm{Zi}$ Wang was supported by the Early Career Faculty grant from NASA's Space Technology Research Grants Program (80NSSC17K0526).

\section{References}

1. E. Kuramochi et al., "Ultrahigh-Q photonic crystal nanocavities realized by the local width modulation of a line defect," Appl. Phys. Lett. 88(4), 041112 (2006).

2. M. Notomi et al., "Waveguides, resonators and their coupled elements in photonic crystal slabs," Opt. Express 12(8), 1551-1561 (2004).

3. T. Tanabe et al., "Trapping and delaying photons for one nanosecond in an ultrasmall high-Q photonic-crystal nanocavity," Nat. Photonics 1(1), 49-52 (2007).

4. D. Englund et al., "Controlling the spontaneous emission rate of single quantum dots in a two-dimensional photonic crystal," Phys. Rev. Lett. 95(1), 013904 (2005).

5. D. Englund et al., "Deterministic coupling of a single nitrogen vacancy center to a photonic crystal cavity," Nano Lett. 10(10), 3922-3926 (2010).

6. D. Englund et al., "Resonant excitation of a quantum dot strongly coupled to a photonic crystal nanocavity," Phys. Rev. Lett. 104(7), 073904 (2010).

7. X. Gan et al., "Strong enhancement of light-matter interaction in graphene coupled to a photonic crystal nanocavity," Nano Lett. 12(11), 5626-5631 (2012).

8. C. Kang et al., "Photonic crystal with multiple-hole defect for sensor applications," Opt. Express 16(22), 18188-18193 (2008).

9. C. Y. Lin et al., "Coupling loss minimization of slow light slotted photonic crystal waveguides using mode matching with continuous group index perturbation," Opt. Lett. 37(2), 232-234 (2012).

10. C. Xiong et al., "Slow-light enhanced correlated photon pair generation in a silicon photonic crystal waveguide," Opt. Lett. 36(17), 3413-3415 (2011).

11. T. Gu et al., "Deterministic integrated tuning of multi-cavity resonances and phase for slow-light in coupled photonic crystal cavities," Appl. Phys. Lett. 98(12), 121103 (2011). 
Xiao et al.: Engineering the light coupling between metalens and photonic crystal resonators...

12. Y. A. Vlasov et al., "Active control of slow light on a chip with photonic crystal waveguides," Nature 438(7064), 65-69 (2005).

13. L. O'Faolain et al., "Low-loss propagation in photonic crystal waveguides," Electron. Lett. 42(25), 1454-1455 (2006).

14. S. Hughes et al., "Extrinsic optical scattering loss in photonic crystal waveguides: role of fabrication disorder and photon group velocity," Phys. Rev. Lett. 94(3), 033903 (2005).

15. Q. V. Tran et al., "Photonic crystal membrane waveguides with low insertion losses," Appl. Phys. Lett. 95(6), 061105 (2009).

16. S. J. McNab et al., "Ultra-low loss photonic integrated circuit with membrane-type photonic crystal waveguides," Opt. Express 11(22) 2927-2939 (2003).

17. F. Wang et al., "Low-loss photonic crystal platform by foundry processing," in CLEO: QELS_Fundam. Sci., Proc., OSA, Paper JTu2D-28 (2020).

18. Y. Xiao et al., "Robust light coupling to photonic crystal waveguide using integrated metalens," in CLEO: Sci. and Innov., Proc., OSA, Paper JTh2B-20 (2020).

19. J. Zhang et al., "Ultrashort and efficient adiabatic waveguide taper based on thin flat focusing lenses," Opt. Express 25(17), 19894-19903 (2017).

20. Y. Fan et al., "Integrated 2D-graded index plasmonic lens on a silicon waveguide for operation in the near-infrared domain," ACS Nano 11(5), 4599-4605 (2017).

21. Z. Wang et al., "On-chip wavefront shaping with dielectric metasurface," Nat. Commun. 10(1), 1-7 (2019).

22. N. Nawi et al., "Enhancement and reproducibility of high quality factor, one-dimensional photonic crystal/photonic wire (1D PhC/PhW) microcavities," J. Eur. Opt. Soc. Rapid. Publ. 14(1), 6 (2018).

23. C. Sauvan et al., "Slow-wave effect and mode-profile matching in photonic crystal microcavities," Phys. Rev. B 71(16), 165118 (2005).

24. C. Sauvan et al., "Modal-reflectivity enhancement by geometry tuning in photonic crystal microcavities," Opt. Express 13(1), 245-255 (2005).

25. Z. Shi, Fundamentals and Applications of Slow Light, University of Rochester (2010).

26. T. D. Happ et al., "Photonic crystal tapers for ultracompact mode conversion," Opt. Lett. 26(14), 1102-1104 (2001).

27. P. Sanchis et al., "Mode matching technique for highly efficient coupling between dielectric waveguides and planar photonic crystal circuits," Opt. Express 10(24), 1391-1397 (2002).

28. G. Pitruzzello et al., "Photonic crystal resonances for sensing and imaging," J. Opt. 20(7), 073004 (2018).

29. Y. Akahane et al., "Fine-tuned high-Q photonic-crystal nanocavity," Opt. Express 13(4), 1202-1214 (2005).

30. Y. Akahane et al., "High-Q photonic nanocavity in a two-dimensional photonic crystal," Nature 425(6961), 944-947 (2003).

31. M. Fujita et al., "Light emission from silicon in photonic crystal nanocavity," IEEE J. Sel. Top. Quantum Electron. 14(4) 1090-1097 (2008).

32. S. Noda et al., "Spontaneous-emission control by photonic crystals and nanocavities," Nat. Photonics 1(8), 449-458 (2007).

33. N. Ismail et al., "Fabry-Pérot resonator: spectral line shapes, generic and related Airy distributions, linewidths, finesses, and performance at low or frequency-dependent reflectivity," Opt. Express 24(15), 16366-16389 (2016).

34. Y. Liu et al., "Real-time dynamic sensing with an on-chip nanophotonic sensor," Opt. Express 25(15), 17201-17210 (2017).

35. N. Skivesen et al., "Photonic-crystal waveguide biosensor," Opt. Express 15(6), 3169-3176 (2007).

36. R. Chandrasekar et al., "Photonic integrated circuits for Department of Defense-relevant chemical and biological sensing applications: state-of-the-art and future outlooks," Opt. Eng. 58(2), 020901 (2019).

Yahui Xiao is a $\mathrm{PhD}$ candidate at the University of Delaware. She received her BS degree from Changchun University of Science and Technology in 2018. Her current research interests include 
Xiao et al.: Engineering the light coupling between metalens and photonic crystal resonators...

photonic crystals and metasurfaces. She is a student member of the Optical Society of America (OSA).

Zi Wang is a $\mathrm{PhD}$ candidate at the University of Delaware. He received his BS and MS degrees from Beijing Institute of Technology in 2014 and 2017, respectively. His current research interests include a multi-layer meta-systems and the application of the integrated photonic devices for machine learning.

Feifan Wang has been a visiting student at the University of Delaware. She received her $\mathrm{PhD}$ from Peking University in 2020. Her current research focuses on on-chip nanophotonic devices based on nonlinear effects.

Hwaseob Lee is a PhD candidate at the University of Delaware. His research interests include several applications of nanophotonics based on silicon microring resonators. He is a student member of OSA.

Thomas Kananen obtained his BS and MS degrees at the University of Delaware in 2018 and 2019, respectively. His research focuses on graphene plasmonic structures in a long wavelength. He is a student member of OSA.

Tingyi Gu is an assistant professor at the University of Delaware. She received her $\mathrm{PhD}$ from Columbia University. Her research focuses on developing passive and active nanophotonic devices on a silicon photonic platform. She is a member of SPIE and OSA. 